\title{
A simple method for characterization of the magnetic field in an ion trap using $\mathrm{Be}^{+}$ions
}

\author{
Jianwei Shen, Andrii Borodin*, and Stephan Schiller* \\ Institut fuer Experimentalphysik, Heinrich-Heine-Universitaet Duesseldorf, 40225 Duesseldorf, Germany \\ ${ }^{*}$ Correspondence addresses: andrii.borodin@uni-duesseldorf.de, step.schiller@uni-duesseldorf.de
}

\begin{abstract}
We demonstrate a simple method for the determination of the magnetic field in an ion trap using laser-cooled ${ }^{9} \mathrm{Be}^{+}$ ions. The method is not based on magnetic resonance and thus does not require delivering radiofrequency (RF) radiation to the trap. Instead, stimulated Raman spectroscopy is used, and only an easily generated optical sideband of the laser cooling wave is required. The d.c. magnetic vector, averaged over the ${ }^{9} \mathrm{Be}^{+}$ion ensemble, is determined. Furthermore, the field strength can be minimized and an upper limit for the field gradient can be determined. The resolution of the method is $0.04 \mathrm{G}$ at present. The relevance for precision rovibrational spectroscopy of molecular hydrogen ions is briefly discussed.
\end{abstract}

\section{Introduction}

Methods of magnetic field measurement are of high importance in different fields of fundamental and applied research - for example in quantum optics, quantum information processing, and high-resolution spectroscopic measurements. The large variety of magnetic field sensors, utilizing e.g. microelectromechanical systems (MEMS), the Hall effect, the giant magnetoresistance effect, superconducting quantum interference (SQUID), have different sensitivities and magnetic field ranges. A common natural disadvantage of these sensors is the need to introduce them into the region where the magnetic field is to be determined. Although many sensors are compact, this requirement becomes critical in the case of, e.g., experiments with trapped ions or atoms, where the presence of vacuum complicates sensor deployment and the sensor can not be used in situ while the experiment is operating. In this case, the trapped ions or atoms themselves can serve as sensors, because the magnetic field affects their level energies, and the measurement of transition frequencies (shifts) can be used to deduce the magnetic field. For an overview of different methods, see e.g. Ref. [1 [3]. Typically, for atoms in the linear Zeeman shift regime, the line splitting is on the order of the Larmor precession frequency, which is $2.8 \mathrm{MHz} / \mathrm{G}$. If a sufficiently large magnetic field can be applied, a RF transition can be used to probe the splitting, see e.g. Ref. [4].

The measurement of a small magnetic field, when the concomitant Zeeman splitting is reduced to on the order of $10 \mathrm{kHz}$ or less, is possible in case of non-zero nuclear magnetic moment and non-zero electronic angular momentum $(J)$, because then RF radiation can be used to measure the splitting between hyperfine states. As an example, the hyperfine transition frequency $f_{0}$ in the ground ${ }^{2} S_{1 / 2}$ level of ${ }^{9} \mathrm{Be}^{+}$is $1.25 \mathrm{GHz}$ in zero field. Using RF spectroscopy between hyperfine states, the frequencies can be measured with $\mathrm{kHz}$ resolution or better. Since the Zeeman shifts of the hyperfine states are on the order of $\mathrm{MHz}$ per Gauss, then a resolution at milli-Gauss level is in principle possible.

Our approach for determining Zeeman shifts of trapped ${ }^{9} \mathrm{Be}^{+}$ions is based on using stimulated Raman transitions. The ${ }^{9} \mathrm{Be}^{+}$ion is a key element of many quantum logic, spectroscopic, and quantum simulation studies [4 7]. The method utilizes only the cooling light and a modulation sideband and does not require delivering a RF field to the trap center, thus simplifying the overall scheme. In fact, no additional hardware beyond the one used for our experiments on sympathetic cooling was employed. 


\section{Motivation}

In our experimental setup, ${ }^{9} \mathrm{Be}^{+}$ions trapped in a linear ion trap are used as coolant ions for sympathetic cooling of co-trapped $\mathrm{HD}^{+}$molecular ions. $\mathrm{HD}^{+}$is a model system in precision spectroscopy of cold trapped molecules for the purpose of testing quantum theory and the time-independence of fundamental constants. Precise measurements of transition frequencies of $\mathrm{HD}^{+}$require information about the magnetic field experienced by the ions in the trap. Therefore, it is natural to consider the coolant ${ }^{9} \mathrm{Be}^{+}$ions as an in-situ magnetic field sensor.

For instance, in pure rotational spectroscopy in $\mathrm{HD}^{+}$at $1.3 \mathrm{THz}$ (see Ref. [8]) it is of interest to resolve the Zeeman structure of individual hyperfine lines. As a concrete example we consider the hyperfine transition $(v=0, N=0, F=0, S=1, J=1) \rightarrow\left(v^{\prime}=0, N^{\prime}=1, F^{\prime}=0, S^{\prime}=1, J^{\prime}=2\right)$ at a detuning $f-f_{0, \text { theor }}=$ $-2.1 \mathrm{MHz}$ from the spinless rotational transition frequency $f_{0, \text { theor }}$ (see Fig. 3 of Ref. [8]). It is particularly attractive, since it exhibits a small Zeeman shift and a small Zeeman splitting. The $J_{z}=0 \rightarrow J_{z}^{\prime}=0$ component of this particular transition has a quadratic Zeeman shift that is approx. $2.9 \mathrm{kHz}$ in a field of $1 \mathrm{G}$. Other metrological properties have been discussed in [9, 10]. Thus, assuming that one can set a magnetic field with the small value $B<0.05 \mathrm{G}$ in the trap, and that the ions are in the Lamb-Dicke regime, a shift and transition linewidth (due to a possible magnetic field gradient) of less than $10 \mathrm{~Hz}$ should be achievable. At this resolution level, a precise measurement of the hyperfine energy contribution $(-2.1 \mathrm{MHz})$ and a test of the QED corrections including those of order $\alpha^{6}$ relative to the nonrelativistic transition frequency would become possible (see Ref. [11]). Note that the closest two Zeeman components arise from transitions $J_{z}= \pm 1 \rightarrow$ $J_{z}^{\prime}= \pm 2$, with nearly opposite linear Zeeman shifts of approx. $\mp 40 \mathrm{kHz} / \mathrm{G}$, respectively, and would be clearly removed from the $J_{z}=0 \rightarrow J_{z}^{\prime}=0$ transition [12]. This example shows the utility of reducing the strength of the magnetic field in an ion trap for a particular application.

\section{Experimental scheme and setup}

The optical transitions in ${ }^{9} \mathrm{Be}^{+}$relevant to laser cooling are shown in Fig. 1. The use of $\sigma^{+}$or $\sigma^{-}$transitions provides an almost closed-cycle level scheme, allowing efficient laser cooling of ${ }^{9} \mathrm{Be}^{+}$ions. In this case, the atomic population is almost completely distributed between the excited ${ }^{2} P_{3 / 2}, F^{\prime \prime}=3, m_{F^{\prime \prime}}= \pm 3$ and the ground ${ }^{2} S_{1 / 2}, F=2, m_{F}= \pm 2$ state; decay into ${ }^{2} S_{1 / 2}, F^{\prime}=1$ state is forbidden, as this would require transitions with $|\Delta m|>1$, which are not allowed in electric-dipole transitions. However, because of non-ideality of the laser polarization state, instability of the magnetic field and its direction, and Larmor precession, the $F^{\prime}=1$ state of the ${ }^{2} S_{1 / 2}$ level becomes populated. An optical sideband ( $\omega_{s}$ in Fig. 1) of the $313 \mathrm{~nm}$ wave used for laser cooling, at $1.25 \mathrm{GHz}$ offset, may be added with the purpose of depleting this state and increase the laser cooling efficiency (repumper).

In this work, we used a two-photon, Doppler-free Raman transition to precisely measure the Zeeman splitting and to determine the magnetic field. In a $\Lambda$-scheme transition, two beams, pump and Stokes $\left(\omega_{c}\right.$ and $\omega_{s}$ ), co-propagate in order to significantly reduce the effect of Doppler broadening, by a factor $\simeq 10^{6}$, so that the residual Doppler linewidth is about $10^{2} \mathrm{~Hz}$.

A phase modulator inserted into the laser beam allows the simple generation of the Stokes wave as a sideband; the fact of simultaneous carrier and sideband generation is important for the stimulated Raman transition. Thus, the light used for the Raman transition is the same as for the purpose of laser cooling and repumping. The stimulated Raman transition becomes strong for a case of the two-photon resonance, i.e. when $\Delta_{C}=\Delta_{S}$, according to the notation in Fig 1. The latter condition is strict for the case of $\Delta_{C}, \Delta_{S} \gg \Omega_{C}, \Omega_{S}$, where $\Omega_{C}$ and $\Omega_{S}$ are the corresponding one-photon Rabi frequencies. Generally, the two-photon transition is spectrally broadened, which is characterized by the effective Rabi frequency for a Raman transition. For our typical conditions, the sideband intensity is varied in the range of a few percent to a few ten percent of the carrier power, and also the total power is varied, and thus the effective Rabi frequency is in the range of few $\mathrm{kHz}$ to many hundred $\mathrm{kHz}$. The a.c. Stark shift is of similar value. Large absolute sideband intensities are conveniently used for preliminary determination of the Raman resonance. Subsequently, a reduction of intensity is necessary for increasing the measurement precision by reducing systematic shifts. 


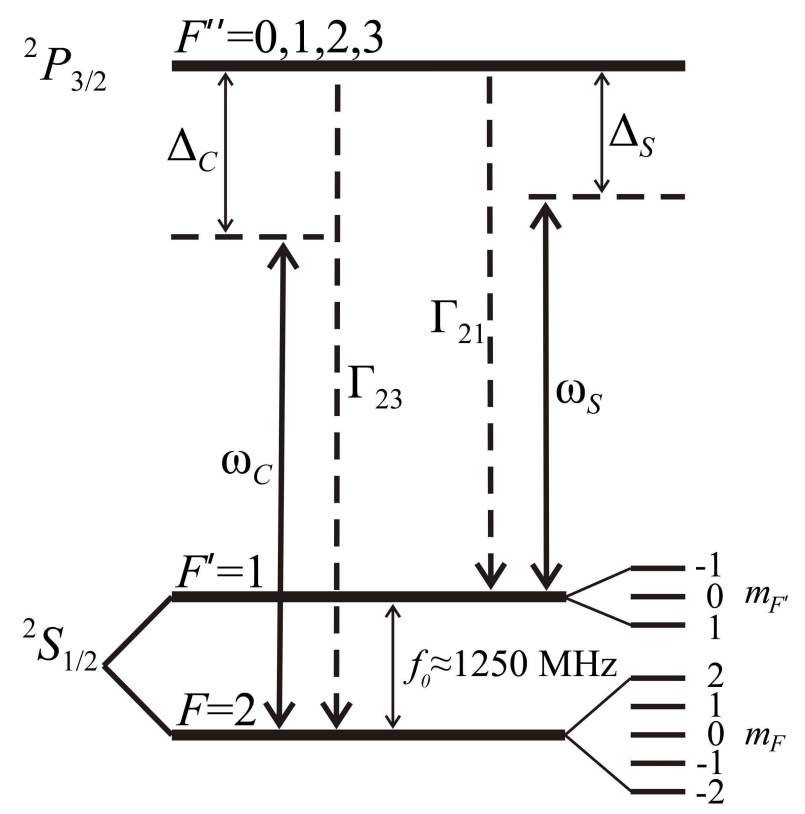

Fig. 1: The optical transitions relevant to the stimulated Raman spectroscopy. $\omega_{C}$ : carrier frequency; $\omega_{S}$ : a red-detuned sideband produced e.g. by an amplitude modulator or phase modulator. Only the Zeeman splittings in the electronic ground state are shown, since those in the electronic excited state are not relevant.

\section{Transitions and level splitting in ${ }^{9} \mathrm{Be}^{+}$}

Stimulated Raman transitions take place between Zeeman states of the hyperfine levels $F=2$ and $F^{\prime}=1$ of the ${ }^{2} S_{1 / 2}$ electronic ground state, with intermediate states being $F^{\prime \prime}=0,1,2$ or 3 of the electronically excited ${ }^{2} P_{3 / 2}$ level. The Zeeman splitting within one hyperfine state is (in the following $F$ refers to any state)

$$
E_{Z}=g_{F} m_{F} \mu_{B} B \simeq 1.4 \frac{\mathrm{MHz}}{\mathrm{G}} g_{F} m_{F} B
$$

where the $g_{F}$ factor is

$$
g_{F} \simeq\left(\frac{3}{2}+\frac{S(S+1)-L(L+1)}{2 J(J+1)}\right) \frac{F(F+1)-I(I+1)+J(J+1)}{2 F(F+1)} .
$$

The possible Zeeman components of the Raman transition $F^{\prime}=1 \rightarrow F=2$ and the corresponding Zeeman shifts (i.e. magnetic-field shift of $\hbar\left(\omega_{c}-\omega_{s}\right)$ ) are:

$$
\begin{array}{lll}
\Delta E_{Z}=(3 / 2,1,1 / 2,0) \times 1.4 \frac{\mathrm{MHz}}{\mathrm{G}} \times B & \text { for } & m_{F^{\prime}}=-1 \rightarrow m_{F}=(-2,-1,0,+1) \\
\Delta E_{Z}=(1,1 / 2,0,-1 / 2,-1) \times 1.4 \frac{\mathrm{MHz}}{\mathrm{G}} \times B & \text { for } & m_{F^{\prime}}=0 \rightarrow m_{F}=(-2,-1,0,+1,+2) \\
\Delta E_{Z}=(0,-1 / 2,-1,-3 / 2) \times 1.4 \frac{\mathrm{MHz}}{\mathrm{G}} \times B & \text { for } & m_{F^{\prime}}=+1 \rightarrow m_{F}=(-1,0,+1,+2)
\end{array}
$$

for $\sigma^{-}, \pi$, and $\sigma^{+}$transitions. Summarizing, the possible Zeeman shifts of the Raman resonance at $1.25 \mathrm{GHz}$ are:

$$
\Delta E_{Z}=(0, \pm 1 / 2, \pm 1, \pm 3 / 2) \times 1.4 \frac{\mathrm{MHz}}{\mathrm{G}} \times B
$$

and a maximum of 7 different Zeeman shifts may be observed.

The excitation spectrum depends on strength of the magnetic field, its direction with respect to the propagation direction of the cooling laser beam and its sideband, on their polarization, and on their intensities. However, we do not attempt to give a complete model, since it is not necessary to do so for the present purpose. 


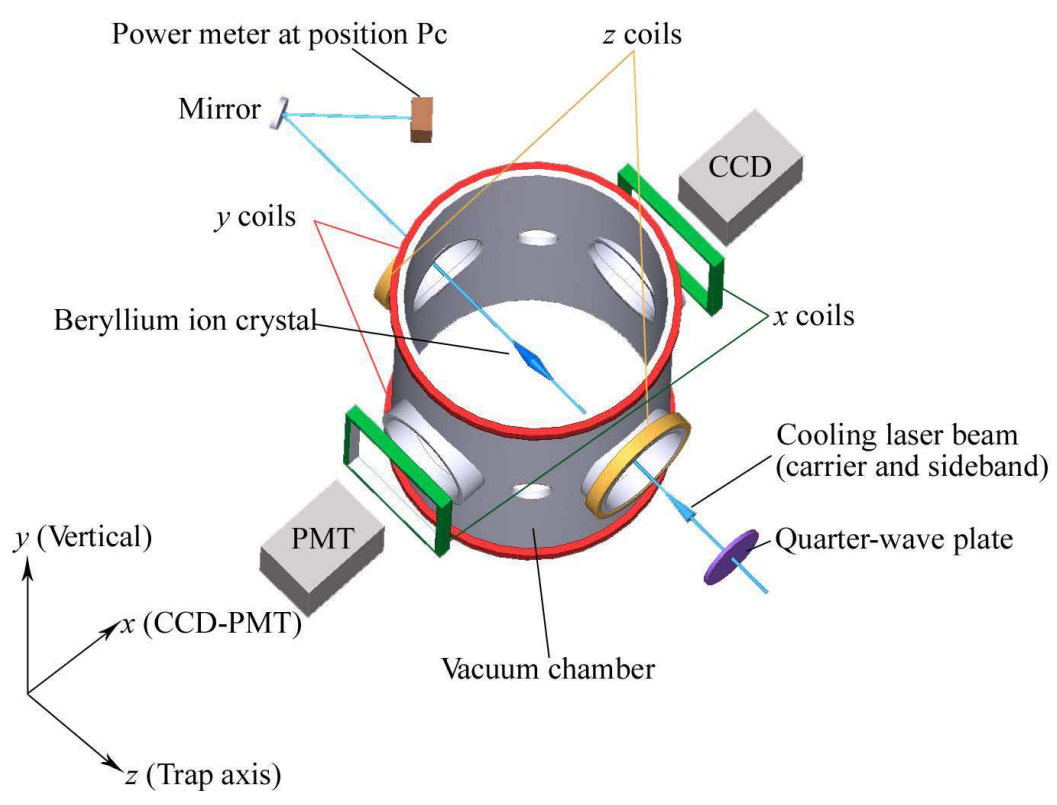

Fig. 2: Geometry of the ion trap apparatus and its magnetic field coils.

It is important to note that a particular transition from the above list Eq. (11) is observed only if the corresponding initial $m_{F^{\prime}}$-state of the $F^{\prime}=1$ hyperfine level is populated. Generally, all $m_{F^{\prime}}$ states can be populated and thus all 7 lines can be detected. There are special cases of $\sigma^{-}$(and $\sigma^{+}$)-induced transitions (carrier and sideband), for which only $m_{F^{\prime}}=-1(+1)$ states of the $F^{\prime}=1$ hyperfine level have significant population in consequence of the interaction with the radiation. As mentioned before, this is caused by Larmor precession, non-ideality of light polarization state, and misalignment of magnetic field vector or field strength fluctuation. The observed Zeeman splittings are then limited to:

$$
\begin{aligned}
& \Delta E_{Z, \sigma^{+}}=-1.4 \frac{\mathrm{MHz}}{\mathrm{G}} \times B, \text { for } \sigma^{+}-\text {induced transition }, \\
& \Delta E_{Z, \sigma^{-}}=+1.4 \frac{\mathrm{MHz}}{\mathrm{G}} \times B, \text { for } \sigma^{-}-\text {induced transition } .
\end{aligned}
$$

\section{Apparatus}

Fig. 2 illustrates the setup used for sympathetic cooling and in which stimulated Raman spectroscopy of ${ }^{9} \mathrm{Be}^{+}$ions has been studied. The cooling laser beam at $313 \mathrm{~nm}$ propagates along the symmetry axis of the linear quadrupole trap ( $z$-direction). We denote the horizontal and vertical directions in the laboratory as $x$ and $y$, respectively. The ${ }^{9} \mathrm{Be}^{+}$fluorescence is partially collected by a CCD camera and by a photomultiplier tube (PMT) operating in the photon counting regime. The latter is used to define the signal. The power of the cooling beam (carrier plus sidebands) is measured after passing the vacuum chamber, at position Pc.

For compensation of the external magnetic field, and for applying a desired magnetic field, three pairs of magnetic field coils are mounted around the vacuum chamber; the directions of the respective fields are nearly orthogonal to each other.

The cooling laser is frequency-tunable and lockable in a detuning range of a few hundred $\mathrm{MHz}$ relative to the ${ }^{9} \mathrm{Be}^{+}$cooling transition. Our laser (see Ref. [13]) includes internally a waveguide electro-optic phase modulator driven at a frequency $f_{\text {mod }} \simeq 1250 \mathrm{MHz}$ that generates a phase modulation sideband pair. The "red" sideband is used as a repumper (wave $\omega_{s}$ in Fig. 11).

${ }^{9} \mathrm{Be}^{+}$ions are loaded and other species are removed from the trap [14]. During this step, the $313 \mathrm{~nm}$ carrier wave is red-detuned by a few hundred $\mathrm{MHz}$ from the cooling transition line, and the sideband power is set to a few ten percent of the carrier power. In order to cool the ${ }^{9} \mathrm{Be}^{+}$ion ensemble and any additional 


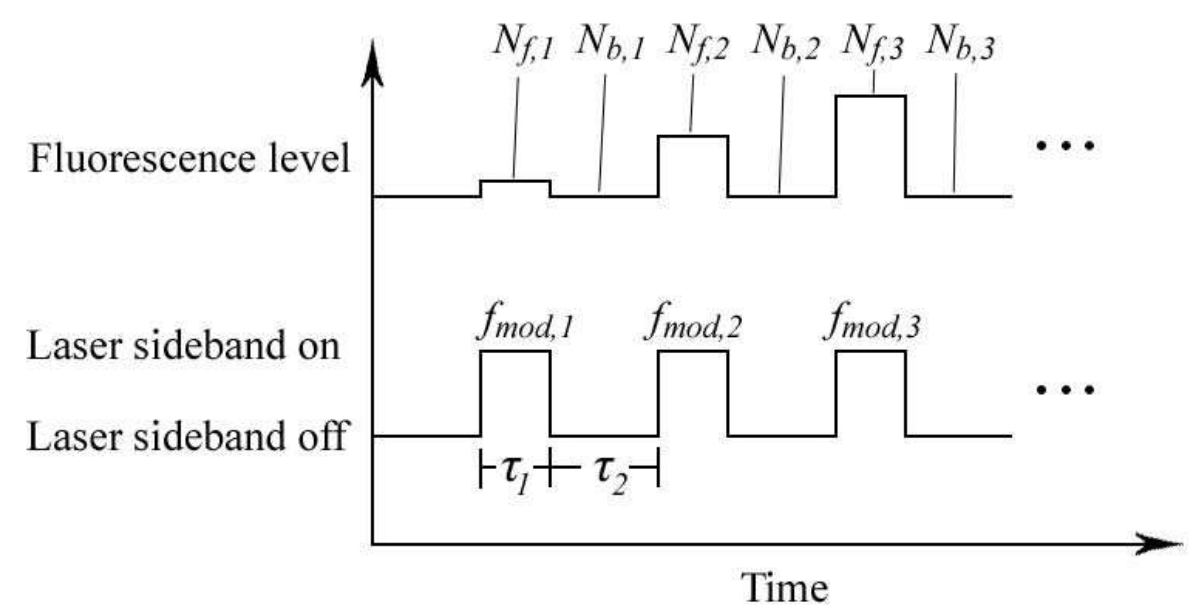

Fig. 3: Measurement time line. See the text for details.

molecular ions to the lowest temperature, or to perform measurements such as the ones described below, the carrier detuning is instead kept at a few ten $\mathrm{MHz}$, and the sideband power is typically reduced by a moderate factor.

For performing stimulated Raman spectroscopy measurements, the sideband frequency $f_{\text {mod }}$ is scanned, while all other parameters are kept constant.

The measurement sequence is illustrated in Fig. 3. During each cycle (duration $\tau_{1}+\tau_{2}$ ) the carrier is always on, while the sideband is turned on and off. During the interval $\tau_{1}$ (sideband on) the PMT signal $N_{f, i}$ is recorded, and during the interval $\tau_{2}$ (sideband off), the signal $N_{b, i}$ is recorded. From one cycle to the next, the sideband frequency is changed from $f_{\bmod , i}$ to $f_{\text {mod,i+1 }}$, in order to scan the transition. For each sequence $i$ of the frequency scan, we define the background-compensated, normalized signal-to-noise ratio as the relative fluorescence $\left(N_{f, i}-N_{b, i}\right) / N_{b, i}$. This removes to some extent the influence of laser power variations, assuming that the background count $N_{b}$ is proportional to laser power (i.e. when it is due to scattered laser light).

In order to minimize the magnetic field in the trap region, an iterative procedure was used. The magnetic field generated by one of the magnetic field coil pairs was modified via its current and the current was optimized so as to produce minimum Zeeman splittings, while the other two field components were kept constant; then the value of the field component of that first coil pair was held constant while the field component generated by the second coil pair was varied, etc. Repeating this procedure several times and also reducing cooling laser power and sideband power in order to achieve higher resolution, we converged to a small magnetic field strength.

\section{Experimental results and data analysis}

In the general case, when all transitions take place, Eq. (1) applies. Fig. 4 illustrates the Zeeman spectrum for a particular, fixed applied magnetic field. Fig. 5] (top) shows the Zeeman splittings for several different values of one magnetic field component, here the $B_{x}$-component. In this measurement it can be directly seen that the transverse magnetic field $B_{y}^{2}+B_{z}^{2}$ is nonzero. For each measurement, the cooling light power was recorded as well.

We also performed measurements similar to the one shown in Fig. 5 but varying the magnetic field component, $B_{y}$ (data set II) and $B_{z}$ (data set III). The data sets I, II, III, all taken at the same power 


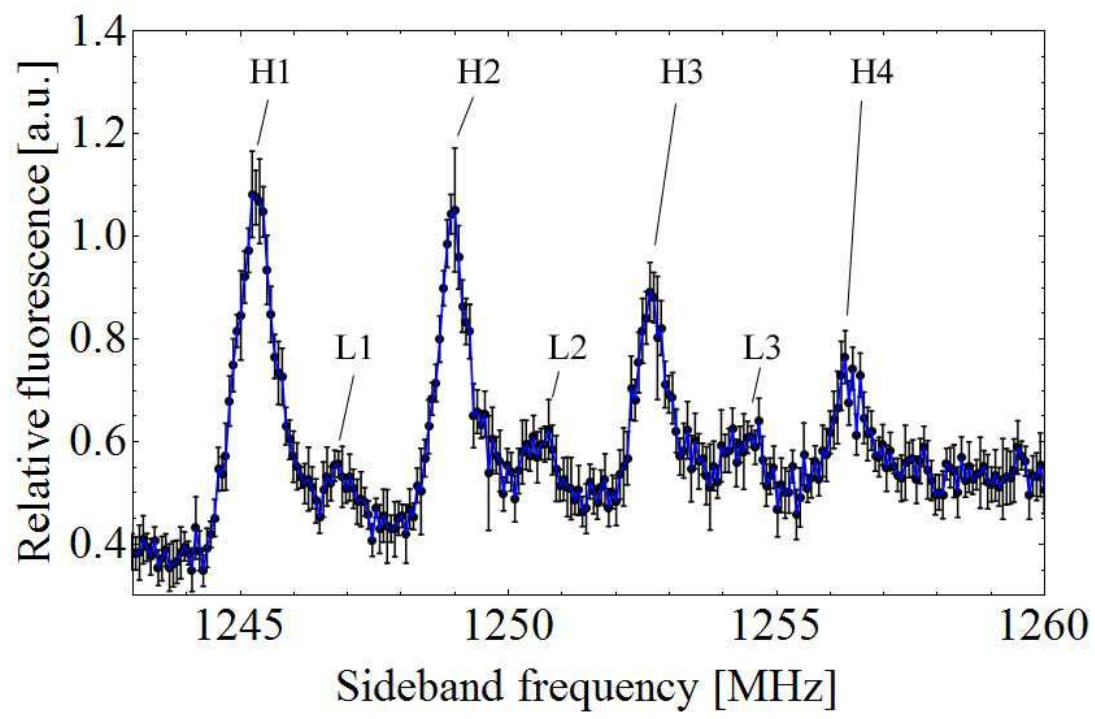

Fig. 4: Zeeman spectrum as a function of sideband frequency $f_{\text {mod }}$. All 7 possible transitions are detected and are denoted H1,..L3. Laser power is $70 \mu \mathrm{W}$. In this measurement, the current settings are $I_{x}=-5.74 \mathrm{~A}, I_{y}=1.70 \mathrm{~A}, I_{z}=0.14 \mathrm{~A}$, which correspond to $B_{x}=-2.434 \mathrm{G}, B_{y}=0.008 \mathrm{G}, B_{z}=$ $1.022 \mathrm{G}$.

(70 $\mu \mathrm{W}$ at position $\mathrm{Pc}$ ), were fitted with the functions:

$$
\begin{aligned}
& f_{\eta}=\eta \sqrt{B_{x}^{2}+B_{y}^{2}+B_{z}^{2}}+f_{\text {offset }}, \\
& B_{j}=k_{j} I_{j}+B_{j, \text { offset }}
\end{aligned}
$$

where $j$ denotes the spatial components $x, y$ and $z$. The values of $\eta$ are taken in accordance with the list Eq. (1). The seven coefficients $B_{j, \text { of fset }}, k_{j}$, and $f_{\text {off set }}$ were obtained from a least-squares fit. The result of this fit may be expressed as:

$$
\begin{aligned}
& B_{x}=(0.362 \pm 0.003) \mathrm{G} / \mathrm{A} \times\left[I_{x}-(0.985 \pm 0.042) \mathrm{A}\right] \\
& B_{y}=(0.434 \pm 0.049) \mathrm{G} / \mathrm{A} \times\left[I_{y}-(1.681 \pm 0.065) \mathrm{A}\right] \\
& B_{z}=(3.586 \pm 0.036) \mathrm{G} / \mathrm{A} \times\left[I_{z}+(0.145 \pm 0.007) \mathrm{A}\right]
\end{aligned}
$$

From this fit, we obtain the current values that minimize the magnetic field components. After setting the components $B_{y}$ and $B_{z}$ close to zero, we recorded data again as a function of $B_{x}$. The result is shown in Fig. 5 (bottom). The effect of the minimization of $B_{y}$ and $B_{z}$ is obvious. Note that in this case, the peaks L1, L2 and L3 are not detected any more.

Subsequently, we set $B_{x}$ and $B_{y}$ close to zero, and varied $B_{z}$. Now the laser wave can be set to $\sigma$ polarization since the magnetic field is then along the direction of propagation. A typical spectrum under a fixed value of $B_{z}$ is shown in Fig. 6. There is only a single resonance. Varying now $B_{z}$, we obtain data set IV, which is displayed in Fig. 7. It was taken with a low value of beam power, $6 \mu \mathrm{W}$ at position Pc.

We also investigated the light-atom interaction effects. The peak shift and the linewidth as a function of beam power are summarized in Fig. 8. The inset of Fig. 8 shows examples of the power dependence of the peak frequency $f_{\eta}$ and of the line shape of one of the two $\sigma$-transitions under near-zero field conditions. We find a linear dependence of the frequency shift and linewidth on the light power. Extrapolation of the peak frequency to zero beam power predicts the hyperfine frequency to be $f_{0}=1250.065 \pm 0.013 \mathrm{MHz}$. A value of $1250017670.46 \pm 1.5 \mathrm{~Hz}$ has been obtained by Nakamura et al [4], and a value of $1250017674.088 \pm 0.024 \mathrm{~Hz}$ has been obtained by Shiga et al [6]; the discrepancy of our measurements from these values $(3.6 \sigma)$ may in part be due to an imperfect polarization state of the beam or the small residual magnetic field. 

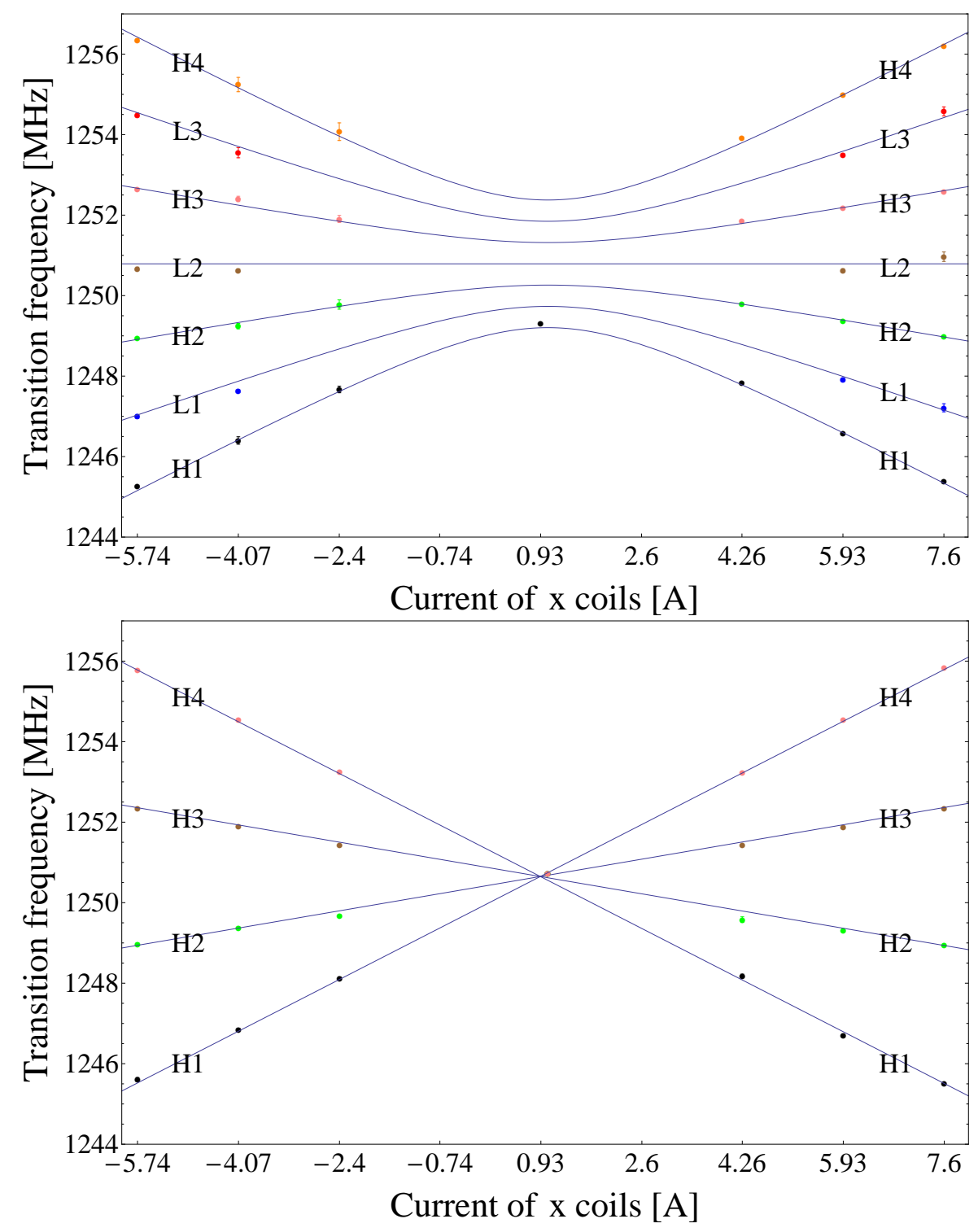

Fig. 5: Zeeman shifts as a function of one magnetic field component, $B_{x}$. Top: Data set I. The data points

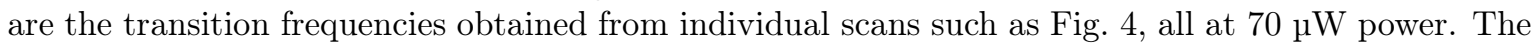
frequencies of that figure are reported in the present plot as data points for the current $I_{x}=-5.74 \mathrm{~A}$. $B_{y}$ and $B_{z}$ are not zero: $B_{y}=0.008 \mathrm{G}, B_{z}=1.022 \mathrm{G}\left(I_{y}=1.70 \mathrm{~A}, I_{z}=0.14 \mathrm{~A}\right)$. Bottom: $B_{y}$ and $B_{z}$ are set to close to zero $\left(I_{y}=1.681 \mathrm{~A}, I_{z}=-0.166 \mathrm{~A}\right)$. 


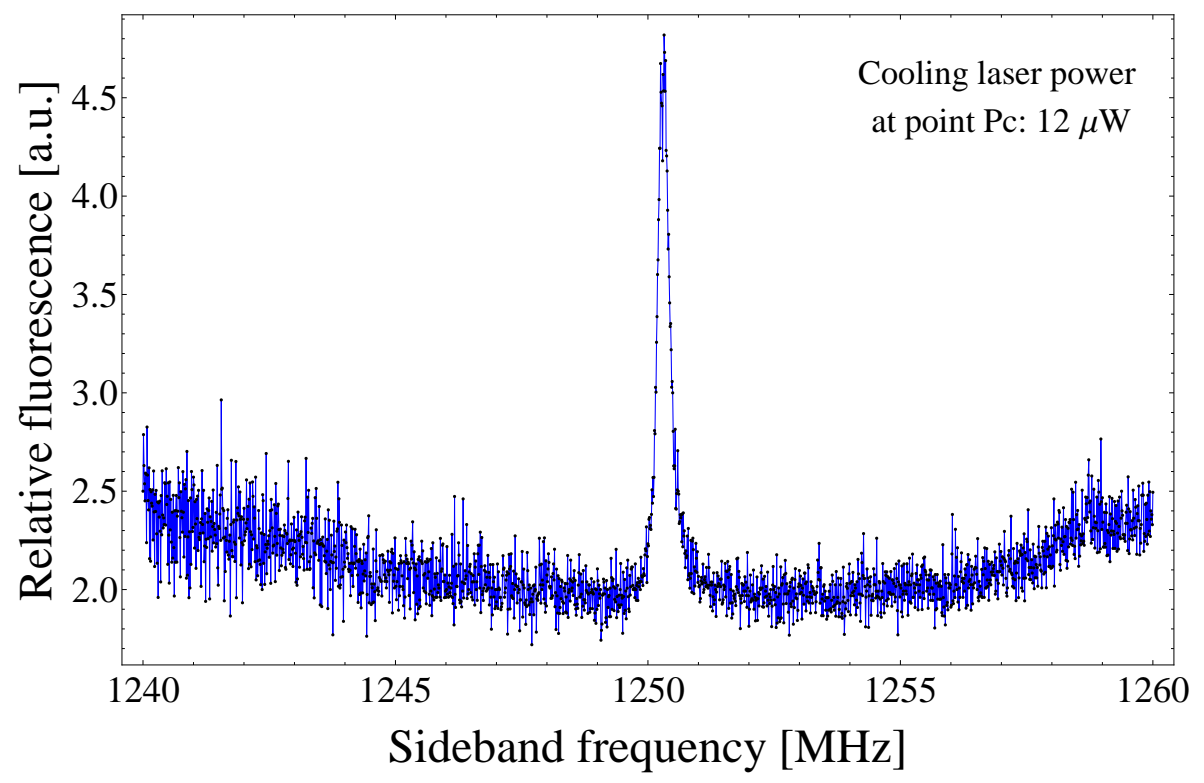

Fig. 6: Spectrum obtained with a $\sigma$-polarized beam. Only one peak is detected. A nonzero magnetic field in the beam propagation direction, $B_{z} \neq 0$, is applied. The other field components are nearly zero.

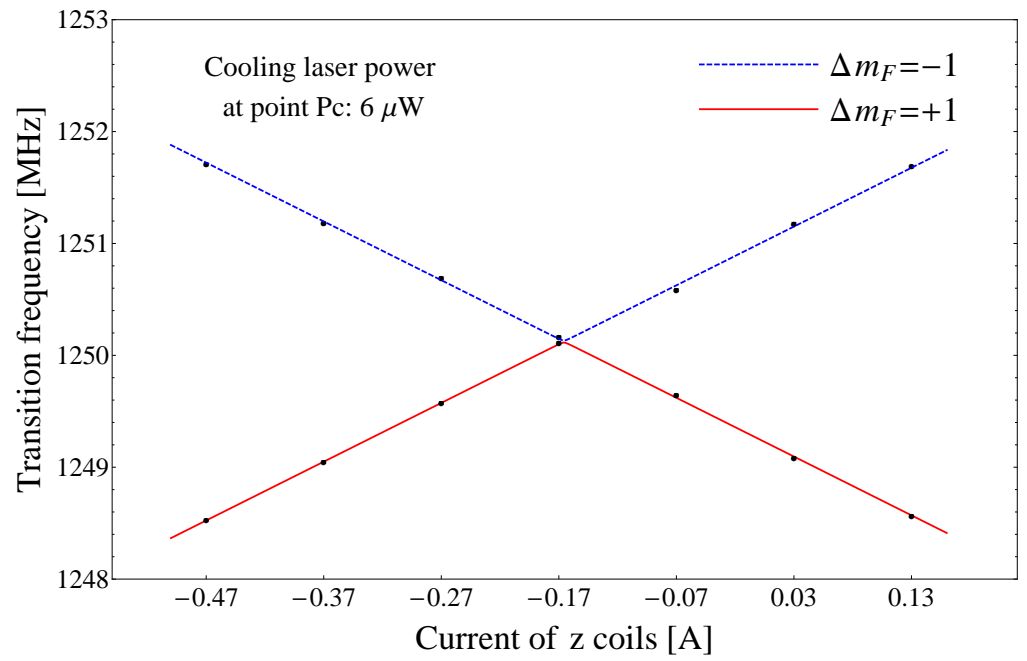

Fig. 7: Zeeman shift of the $\Delta m_{F}=m_{F^{\prime}}-m_{F}=\mp 1$ transitions as a function of $B_{z}$. The magnetic field components $B_{x}$ and $B_{y}$ are minimized. 


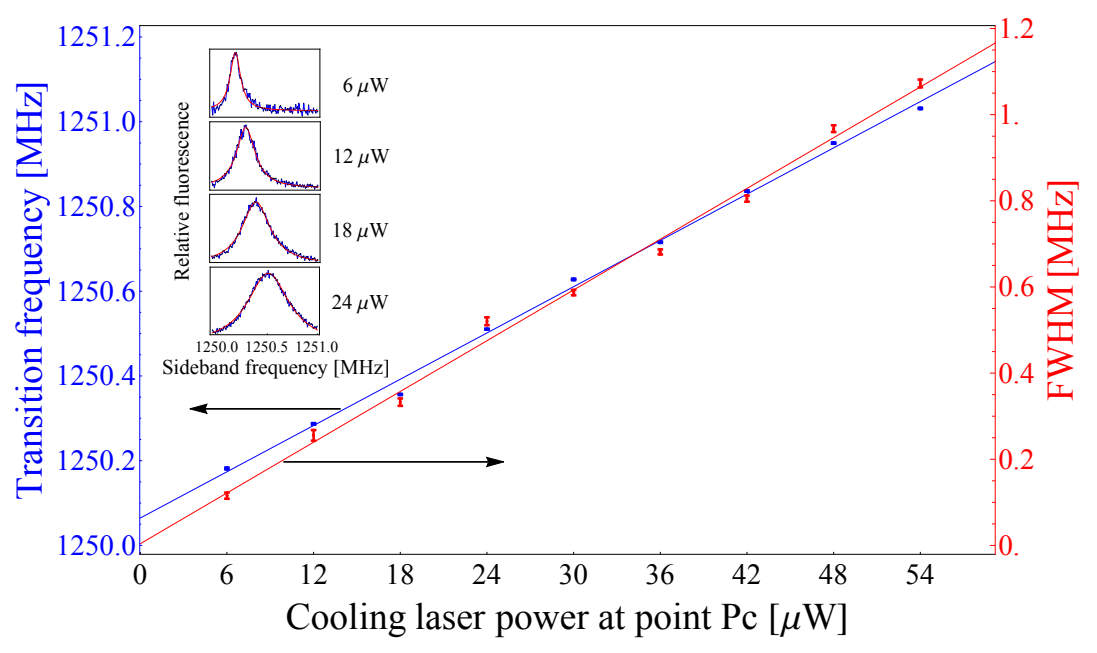

Fig. 8: Light shift and power broadening as a function of laser power. Inset: light shift and power broadening of a $\sigma$-transition. All spectra were taken under the same conditions, only the beam power was varied. The magnetic field strength is nearly zero.

\section{Discussion}

In this work, we focus on the ability to minimize the magnetic field. We consider this issue from two points of view. First, the fit of data sets I, II, III allows us to compute the magnetic field uncertainty when the currents are set to minimize the field components. The result is

$$
\Delta B=\sqrt{(0.362 \times 0.042)^{2}+(0.434 \times 0.065)^{2}+(3.586 \times 0.007)^{2}} \mathrm{G}=0.041 \mathrm{G} .
$$

We can also consider the data set IV (Fig. 7). A fit of only this data yields

$$
\begin{aligned}
B_{z} & =(3.753 \pm 0.020) \mathrm{G} / \mathrm{A} \times\left[I_{z}+(0.166 \pm 0.001) \mathrm{A}\right] \\
\sqrt{B_{x}^{2}+B_{y}^{2}} & =(0.007 \pm 0.031) \mathrm{G} .
\end{aligned}
$$

The result for the current value that minimizes $B_{z}$ lies outside the $2 \sigma$ uncertainty range of the result obtained in Eq.(4), but it should be noted that the conditions of data set IV were different: lower beam power and smaller range of offsets from the zero-field-component current value. Nevertheless, the field strength uncertainty, $\Delta B^{\prime}=\sqrt{(3.753 \times 0.001)^{2}+(0.031)^{2}} \mathrm{G}=0.031 \mathrm{G}$ is comparable to $\Delta B$.

As a third result, we can consider the measurement performed with near-zero field, and consider the difference between the zero-intensity extrapolated resonance frequency of Fig. 8 and the literature experimental value, $\Delta f_{0}=47 \pm 13 \mathrm{kHz}$. From this we may set an upper limit to the field strength present during that measurement, $(47+13) \mathrm{kHz} /(1.4 \mathrm{MHz} / \mathrm{G}) \simeq 43 \mathrm{mG}$.

Finally, we can provide an upper limit for the magnetic field gradient as follows. The narrowest linewidth observed when reducing the laser power as much as possible while keeping the ions cooled (approximately $2 \mu \mathrm{W}$ ), is approximately $40 \mathrm{kHz}$. Assigning the linewidth to be due completely to a nonzero magnetic field gradient across the ion ensemble (size: $2 \mathrm{~mm}$ in $z$-direction), it would then be equal or less to $40 \mathrm{kHz} /(1.4 \mathrm{MHz} / \mathrm{G}$ ) $\simeq 29 \mathrm{mG}$. This is a conservative upper limit, since Fig. 8 indicates that at this laser power there is still lightinduced broadening present.

\section{Conclusion and outlook}

We demonstrated a simple method for measuring the magnetic field in an ion trap that uses beryllium ions. The method is simple since it utilizes only the cooling laser and an optical sideband that is also 
conventionally present since it serves as a repumper during laser cooling. The magnetic field resolution, approximately $40 \mathrm{mG}$, is at present limited by power broadening and the Stark shift induced by the cooling wave, imperfect polarization state of the cooling wave, and by the temporal instability of the currents supplied to the magnetic field coils (here, the instability is of the order of $1 \mathrm{~mA}$ ). In addition, a non-uniformity of the magnetic field, if present, also limits the resolution of the absolute field strength determination.

The method has been applied here to an ensemble containing a large number of trapped ions (a few thousands), since this is typical for our application of sympathetic cooling. We believe that the method could be applied also in the case of few ions or a single trapped ion. In the case of a moderate number of trapped ions (e.g. an ion string), the use of a tightly focused beam would allow addressing an individual ion and determining the field at its location.

As a further perspective, we propose that it may be possible to eliminate the light shift. This requires equal Rabi frequencies of the sideband and the carrier; then the light shifts induced by the two radiation fields compensate each other. The one-photon transition induced by the strong sideband, can, in principle, hinder from observation the two-photon transition, but an appropriate choice of intensities and detunings can allow for (partial) compensation of the light shifts and for detection of the two-photon signal. A complete compensation of the frequency shifts means equality of sideband and carrier intensities (neglecting for simplicity the issue of branching ratios). Thus, this scheme requires a modulation index of about 0.33 , which is available from high-efficiency waveguide modulators.

Acknowledgement: We are indebted to Q.-F. Chen for assistance in the data analysis. We are also grateful to M. Hansen and U. Bressel for performing a magnetic field characterization using magnetic sensors. This work was done in the framework of project Schi 431/19-1 funded by the Deutsche Forschungsgemeinschaft.

\section{References}

[1] S. A. Webster, P. Taylor, M. Roberts, G. P. Barwood, and P. Gill, Phys. Rev. A 65, 052501 (2002).

[2] T. Schneider, E. Peik, and C. Tamm, Phys. Rev. Lett. 94, 230801 (2005).

[3] T. Schneider, Optical frequency standard with a single ${ }^{171} Y b^{+}$ion, Ph.D. thesis, University Hannover (2005).

[4] T. Nakamura, M. Wada, K. Okada, I. Katayama, S. Ohhtani, and H. A. Schuessler, Optics Communications 205, 329 (2002).

[5] C. Chou, D. B. Hume, J. C. J. Koelemeij, D. J. Wineland, and T. Rosenband, Phys. Rev. Lett. 104, $070802(2010)$.

[6] N. Shiga, W. Itano, and J. Bollinger, Phys. Rev. A 84, 012510 (2011).

[7] M. Biercuk, H. Uys, A. VanDevender, N. Shiga, W. Itano, and J. Bollinger, Nat. Letters 458, 996 (2009).

[8] J. Shen, A. Borodin, M. Hansen, and S. Schiller, Phys. Rev. A 85, 032519 (2012).

[9] D.Bakalov and S.Schiller, Appl. Phys. B 114, 213 (2014).

[10] S. Schiller, D. Bakalov, and V. I. Korobov, Phys. Rev. Lett. 113, 023004 (2014).

[11] V. I. Korobov, L. Hilico, and J. P. Karr, Phys. Rev. A 89, 032511 (2014).

[12] D. Bakalov, V. Korobov, and S. Schiller, J. Phys. B: Al. Mol. Opt. Phys. 44, 025003 (2011), corrigendum: J. Phys. B: Al. Mol. Opt. Phys., 45, 049501 (2012).

[13] S. Vasilyev, A. Nevsky, I. Ernsting, M. Hansen, J. Shen, and S. Schiller, Appl. Phys. B 103, 27 (2011).

[14] P. Blythe, B. Roth, U. Frohlich, H. Wenz, and S. Schiller, Phys. Rev. Lett. 95, 183002 (2005). 